\title{
Harmonic angular Doppler effect
}

\author{
By making use of the spin angular momentum of light, rotational frequency shifts of harmonic waves generated by \\ spinning nonlinear media have been observed.
}

\section{Etienne Brasselet}

A frequency shift experienced by an observer whenever a source of waves moves away from or towards the

observer is a feature common to all waves a phenomenon called the Doppler effect. Indeed, everyday examples are sufficiently abundant to guarantee that everyone has experienced the Doppler effect, most probably as sound waves whose pitch is higher or lower than that of the original source (emitting at a fixed frequency in its own reference frame) depending on whether the source moves towards or away from the listener. A different common example is that of ripples (capillary waves) formed at the surface of water around a moving object. The measurement of frequency or wavelength shifts can thus be turned into velocity data, which finds various applications, for instance in medicine, where non-invasive quantitative blood flow analysis via the acoustic Doppler effect guides clinical decisions in many circumstances.

The effect is, of course, not limited to acoustic waves. Doppler frequency shift of radio and optical electromagnetic waves is commonly exploited for automobile speed detection. In fact, it is optical frequency shifts that Christian Doppler used as his primary example in the middle of the nineteenth century to explain the colour of binary stars. The optical Doppler effect is ubiquitous to light-matter interaction and its signature is found when looking at both atoms or at distant galaxies. Still, although the Doppler effect usually implies moving media involving spatial translations, this is not always the case. In fact, there is a variant to the 'translational' Doppler effect - the so-called rotational Doppler effect that leads to frequency shifts even if the distance between the source and the observer is fixed. Analogously, this can be understood in terms of relative angular motion between the source and an observer, which implies that the spin and/or orbital angular degree of freedom of light are at play. An early experimental observation based on spin angular momentum was reported 50 years ago using circularly polarized microwaves ${ }^{1}$ and frequency shift due to orbital angular momentum of light dates back almost 20 years (ref. 2 ).
Now, a collaboration between scientists from the University of Birmingham in the UK and the University of Paderborn in Germany reports on the experimental observation of a rotational frequency shift during second-harmonic generation, a nonlinear optical phenomenon in which the frequency of a fundamental wave is doubled via nonlinear interaction with a noncentrosymmetric medium ${ }^{3}$. Interestingly, the experiment unveils a frequency shift that differs from that usually obtained in the linear optics regime.

The researchers had to solve a number of practical issues to reach their goal. The first step was to identify an appropriate nonlinear optical medium. Because the rotational Doppler shift implies the presence of optical angular momentum, an ideal situation corresponds to two photons of a circularly polarized fundamental (F) plane wave with wavelength $\lambda_{\mathrm{F}}$ generating one collinear photon of a circularly polarized second-harmonic ( $\mathrm{SH}$ ) plane wave with wavelength $\lambda_{\mathrm{SH}}$. Then, with the knowledge of selection rules pertaining to harmonic generations with circularly polarized light, Li et al. selected a nonlinear crystal with three-fold rotational symmetry (a commercially available $\beta$-barium borate crystal) whose optical axis is aligned along the propagation direction of light. Indeed, in such a situation, a fundamental wave with helicity $\sigma_{\mathrm{F}}= \pm 1$ (the sign refers to the handedness of the circular polarization state) selectively produces a secondharmonic wave with flipped helicity $\sigma_{\mathrm{SH}}=-\sigma_{\mathrm{F}}$. That said, the textbook picture of the situation with the nonlinear crystal at rest is expressed as

$$
\lambda_{\mathrm{SH}}=\lambda_{\mathrm{F}} / 2
$$

The second step involved an experimental set-up encompassing the relative rotating motion of the harmonic wave and an observer, and to achieve this the nonlinear crystal is rotated at constant angular frequency $\Omega$ around its axis of symmetry. The experiment requires detection of a tiny harmonic wavelength change $\delta \lambda=\lambda_{\mathrm{SH}}-\lambda_{\mathrm{F}} / 2$ in the laboratory frame in presence of the rotational Doppler effect, as shown in Fig. 1.
Introducing the harmonic angular frequency change $\delta \omega=\omega_{\mathrm{SH}}-2 \omega_{\mathrm{F}}$, Li et al. ${ }^{3}$ derived the expected Doppler shift as

$$
\delta \omega=-3 \sigma_{\mathrm{F}} \Omega
$$

From the experimental parameters used, namely fundamental wavelength $\lambda_{\mathrm{F}}=1,100 \mathrm{~nm}$ and crystal rotation frequency of the order of a few tens of Hertz, one obtains from the relationship $\delta \lambda / \lambda_{\mathrm{SH}}=-\delta \omega / \omega_{\mathrm{SH}}$ :

$$
|\delta \lambda|=\frac{3 \sigma_{\mathrm{F}} \Omega \lambda_{\mathrm{F}}^{2}}{8 \pi c} \approx 10^{-19} \mathrm{~m}
$$

an extremely small value that could dampen experimental enthusiasm ( $c$ is the speed of light). However, the scientists used a wellknown trick in the field of optical Doppler frequency shift measurements - they measured the frequency change given by equation (2) instead of evaluating the tiny distance change given by equation (3). This frequency change measurement is made easily by interfering the frequency-shifted second-harmonic wave at the output of the rotating nonlinear crystal with a secondharmonic wave generated from a static identical nonlinear crystal. Because the interfering waves have similar frequency, their temporal beating is at a low angular frequency $\delta \omega$, whose detection is relatively easy despite the small amplitude of the timeperiodic second-harmonic intensity signal.

Indeed, the nonlinear process efficiency is measured to be as small as less than one part per million for the 5-mm-long $\beta$-barium borate nonlinear crystal used that was irradiated by a fundamental femtosecond laser beam with $82-\mathrm{MHz}$ repetition rate, 200 -fs pulse duration and 30-mW average power. Importantly, it is experimentally demonstrated that the observed frequency shift is independent of the wavelength, scales linearly with the rotation rate of the nonlinear crystal and changes its sign both with the helicity of the fundamental wave and the nonlinear crystal rotation direction, thus fully validating equation (2). 
It could be argued that the derivation by Li et al. ${ }^{3}$ for the frequency shift given by equation (2) (equation (5) of ref. 3) does not strictly follow the experimental scenario of a nonlinear crystal rotating at fixed angular frequency $\Omega$ but instead introduces two distinct rotational frequencies, namely those before and after the second-harmonic generation process takes place with two incident photons of the fundamental waves. It is interesting to point out that constant $\Omega$ means that that power is extracted from or added to (depending on the relative rotation direction between the fundamental-wave electric field and the nonlinear crystal, that is, the sign of $\sigma_{\mathrm{F}} \Omega$ ) the fundamental wave by the external motor as part of the generation of higher-harmonic ( $\mathrm{HH}$ ) wave of order $n$ and helicity $\sigma_{\mathrm{HH}}$. Energy conservation thus implies that the power produced by light in presence of nonlinear crystal rotation is

compensated by angular frequency shift $\delta \omega$ of a higher-harmonic wave:

$$
\Gamma_{\mathrm{opt}} \Omega+\frac{P \delta \omega}{n \omega_{\mathrm{F}}}=0
$$

where $P$ is incident average power and $\Gamma_{\text {opt }}$ is the amplitude of the optical torque (directed along the propagation direction) exerted on the nonlinear crystal that can be derived by evaluating the optical spin angular momentum transfer to matter, as each photon of a circularly polarized wave with helicity $\sigma$ carries $\sigma \hbar$ angular momentum along the propagation direction ( $\hbar$ is the reduced Planck constant):

$$
\Gamma_{\text {opt }}=\left(\sigma_{\mathrm{F}} n-\sigma_{\mathrm{HH}}\right) \frac{P}{n \omega_{\mathrm{F}}}
$$

Combining equations (4) and (5) above, one obtains equation (4) of ref. 3 while preserving the condition of a constant $\Omega$, which is expressed with the present notation as

$$
\delta \omega=-\left(\sigma_{\mathrm{F}} n-\sigma_{\mathrm{HH}}\right) \Omega
$$

It is worth mentioning that the experimental manifestation of a rotational Doppler effect was demonstrated 20 years ago in a nonlinear optics experiment ${ }^{4}$ by irradiating a thin film (tens of micrometres thick) of liquid crystals by a continuouswave circularly polarized laser beam. However, a few differences between this experiment and the one reported by $\mathrm{Li}$ et al. should be emphasized. First, the rotational Doppler effect reported in ref. 4 results from a self-induced nonlinear phenomenon, the nonlinear medium being rotated by the light itself. Such a situation would hardly be observable in the approach of Li et al. even though the optical torque exerted on the medium per incident 'working photon' is larger than in ref. 4 by a factor $3 / 2$, because of the extremely low

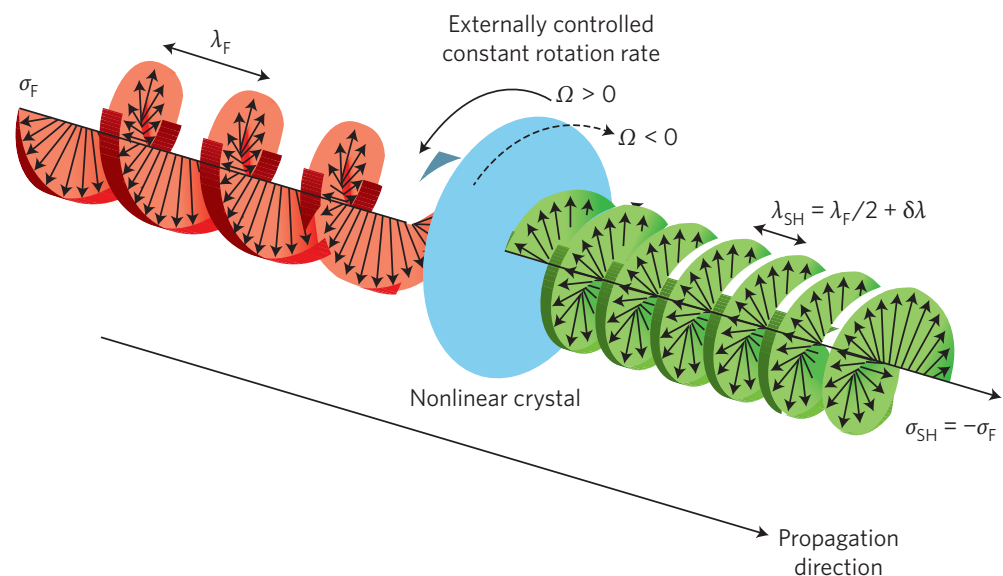

Figure 1 | Principle of the rotational Doppler shift experiment performed by Li and colleagues. A spinning nonlinear crystal with three-fold rotational symmetry is rotated at constant angular frequency $\Omega$ around its optical axis. $\lambda_{i}$ and $\sigma_{i}$, with $i=(\mathrm{F}, \mathrm{SH})$, refer to wavelength and helicity of the fundamental (F) and second-harmonic ( $\mathrm{SH}$ ) circularly polarized waves, and $\delta \lambda$ (given by equation (3)) refers to tiny wavelength change arising from the rotational Doppler effect. Depicted helices correspond to the spatial distribution of the electric field of the fundamental and second-harmonic wave at a given time (here for $\sigma_{\mathrm{F}}=-1$ ). Transmitted fundamental wave is not shown for clarity.

efficiency of the nonlinear process mentioned above and the macroscopic size of the nonlinear crystal. Second, the nature of the nonlinear process is orientational in ref. 4 and does not involve the generation of a new wave, whereas the electronic nonlinearity at play in the work of Li et al. leads to the generation of a secondharmonic wave.

Remarkably, the generation of additional waves via electronic nonlinearities that are not restricted to quadratic nonlinearity as experimentally explored by $\mathrm{Li}$ et al., provides access to enhanced rotational Doppler shifts ${ }^{3}$. This could lead to the development of contactless, sensitive, nonlinear optical detection of rotational motions, although the signal level is an issue that remains to be solved before practical implementation involving higher harmonics is possible. Accordingly, it would be interesting to explore whether a quasi-phase-matching strategy, a welldeveloped technique in laser physics that involves spatial modulation of the material nonlinearity to obtain highly efficient harmonic generation, could be implemented.

Only the spin contribution to the optical angular momentum has been mentioned so far, thus an open question is related to the possible role of the orbital angular momentum. In particular, spin-orbit interaction of light, which couples the polarization degree of freedom to the spatial degrees of freedom of light fields $s^{5}$, may lead to enhancement of the rotational Doppler effect. To this end, an option building on the scheme used by Li et al., where light propagates along the optical axis of a crystal with discrete rotational symmetry, could be to enrich the angular spectrum content of the fundamental field. Indeed, previous works considering nonlinear harmonic generation of Bessel beams ${ }^{6}$ suggest intriguing interplay between spin and orbital angular momentum of light. Finally, it would also be interesting to explore the generic features of the rotational Doppler effect by extending the proposed approach to other kinds of waves.

Etienne Brasselet is at the University of Bordeaux and CNRS, LOMA, UMR 5798, F-33400 Talence, France.

e-mail: etienne.brasselet@u-bordeaux.fr

References

1. Allen, P. J. Am. J. Phys. 34, 1185-1192 (1966)

2. Courtial, J., Dholakia, K., Robertson, D. A., Allen, L. \& Padgett, M. J. Phys. Rev. Lett. 80, 3217-3219 (1998).

3. Li, G., Zentgraf, T. \& Zhang, S. Nature Phys. http://dx.doi. org/10.1038/nphys3699 (2016).

4. Santamato, E., Daino, B., Romagnoli, M., Settembre, M. \& Shen, Y. R. Phys. Rev. Lett. 57, 2423-2426 (1986).

5. Bliokh, K. Y., Rodriguez-Fortuno, F. J., Nori, F. \& Zayats, A. V. Nature Photon. 9, 796-808 (2015).

6. Belyi, V. N., Khilo, N. A., Forbes, A. \& Ryzhevich, A. A. Proc. SPIE 7430, 74300F (2009). 